ORIGINAL RESEARCH

\title{
Sildenafil and Bosentan Improve Arterial Oxygenation During Acute Hypoxic Exercise: A Controlled Laboratory Trial
}

\author{
I. Mark Olfert, PhD; Alexander Loeckinger, MD; Benedikt Treml, MD; Martin Faulhaber, PhD; \\ Markus Flatz, PhD; Martin Burtscher, MD, PhD; Susanne Truebsbach, MD; Axel Kleinsasser, MD \\ From the Center for Cardiovascular and Respiratory Sciences, Division of Exercise Physiology, West Virginia University School of Medicine, \\ Morgantown, WV (Dr Olfert); Section of Respiratory and Altitude Research, Department of Anesthesiology and Critical Care Medicine, \\ Innsbruck Medical University, Innsbruck, Austria (Drs Loeckinger, Treml, Truebsbach, and Kleinsasser); Department of Sports Sciences, \\ Leopold-Franzens University, Innsbruck, Austria (Drs Faulhaber and Burtscher).
}

\begin{abstract}
Objectives.-Sildenafil and, recently, bosentan have been reported to increase arterial saturation and exercise capacity at altitude. The mechanisms behind this are still poorly defined but may be related to attenuation of hypoxic pulmonary vasoconstriction (HPV) and improved gas exchange. This study was designed to examine and compare the effect of sildenafil and bosentan on pulmonary gas exchange during acute hypoxic exercise in a controlled laboratory setting.

Methods.-Sixteen athletic university students ( 8 males, 8 females) were examined during exercise in a hypoxic chamber $(11 \%$ oxygen) before and after the administration of either sildenafil $(\mathrm{n}=10)$ or bosentan $(n=6)$. Respiratory and metabolic measurements were taken at rest and during increasing exercise intensity (up to $90 \%$ of their individual maximal oxygen uptake $\left[\mathrm{V}_{2} \max \right]$ ) in concert with arterial blood gas sampling.

Results.-Both drugs resulted in small, but significant increases in arterial $\mathrm{PO}_{2}\left(2-3\right.$ Torr) and $\mathrm{O}_{2}$ saturation (3-4\%) at rest and during hypoxic exercise, in both men and women. No significant changes in arterial $\mathrm{PCO}_{2}$ or ventilation were seen at rest or during exercise in hypoxia; however, heart rate (both at rest and during exercise) was increased with both sildenafil and bosentan in both men and women.

Conclusions.- These data demonstrate that sildenafil and bosentan equally improve arterial oxygenation in acute hypoxia in both men and women, which could account for improved physical performance at altitude.
\end{abstract}

Key words: phosphodiesterase-5 inhibitor, endothelin receptor antagonist, gas exchange

\section{Introduction}

Sojourners to high altitude $(>3000 \mathrm{~m})$ typically experience hypoxemia and exercise intolerance. Under resting conditions, the alveolar-arterial $\mathrm{PO}_{2}$ difference $\left(\mathrm{A}-\mathrm{aDO}_{2}\right)$ is often unchanged (or normal) at high altitude, indicating that hypoxemia is due to reduced inspired $\mathrm{PO}_{2}$ without impairment in gas exchange. However, even during light physical activity at high altitude, the $\mathrm{A}-\mathrm{aDO}_{2}$ becomes widened due to $\mathrm{O}_{2}$ diffusion limitation and greater ventilation/perfusion mismatching

Corresponding author: I. Mark Olfert, PhD, Center for Cardiovascular and Respiratory Sciences, West Virginia University School of Medicine, Division of Exercise Physiology, PO Box 9105, Morgantown, WV 26506 (e-mail: molfert@hsc.wvu.edu). that progressively worsens with increasing work intensity. ${ }^{1-3}$ Pulmonary arterial hypertension and subclinical pulmonary interstitial fluid accumulation are among the mechanisms believed to be responsible for ventilation/ perfusion mismatching and $\mathrm{O}_{2}$ diffusion limitation during hypoxic exercise. ${ }^{1-4}$

It has long been recognized that exposure to high altitude results in hypoxic pulmonary vasoconstriction (HPV), which is characterized by increased pulmonary arterial pressure (PAP) and greater pulmonary vascular resistance (PVR) due to hypoxia-mediated smooth muscle contraction in the pulmonary vasculature. In pathological conditions, where alveolar hypoxia may occur in localized lung regions, HPV is a useful adaptive mechanism to divert blood flow away from poorly ventilated 
Table 1. Effect of phosphodiesterase-5 inhibition on exercise performance

\begin{tabular}{|c|c|c|c|c|c|c|}
\hline & \multirow[b]{2}{*}{ No. of subjects } & \multirow[b]{2}{*}{ Acute hypoxia $<2$ hours } & \multicolumn{4}{|c|}{ Days at Altitude } \\
\hline & & & 1 & 2 & 5 & 10 \\
\hline Ghofrani et al (2004) & 14 & & +++ & - & & \\
\hline Myers et al (2004) & 15 & & + & & & \\
\hline Richalet et al (2005) & 6 & & & ++ & ++ & \\
\hline Hsu et al (2006) & 11 & ++ & & & & \\
\hline Faoro et al (2007) & 16 & ++ & & & & - \\
\hline
\end{tabular}

Each "+" symbol represents improved exercise perfomance compared to placebo group.

$+=<10 \%$ improvement, $++=10-20 \%$ improvement, $+++=20-30 \%$ improvement, $-=$ no change.

lung areas and preserve pulmonary blood flow to wellventilated areas of the lung. In contrast, at high altitude (where the entire lung is hypoxic), excessive HPV can be detrimental, resulting in increased PAP and leading to high altitude pulmonary edema (HAPE) — a condition that impairs gas exchange because of fluid accumulation in the alveoli and may be fatal if left untreated. ${ }^{5-7}$ Moreover, increased pulmonary arterial resistance may limit exercise capacity by limiting right ventricular afterload.

Sildenafil is a selective phosphordiesterase-5 inhibitor that dilates the pulmonary vascular bed by increasing intracellular guanosine 3',5'-cyclic monophosphate (cGMP), relaxing vascular smooth muscle in pulmonary arterioles, and has been shown to be beneficial in the treatment of patients with primary pulmonary arterial hypertension. ${ }^{8-11}$ Similarly, bosentan, a nonselective endothelin receptor antagonist preventing endothelin-1-triggered vasoconstriction in the lung, is also used in treating patients with primary pulmonary arterial hypertension. ${ }^{12-14}$ Recently, a single oral dose of bosentan has also been shown to reduce hypoxia-induced increases in PAP in healthy individuals exposed to acute hypoxia. ${ }^{15}$ Both drugs have been shown to improve exercise capacity in patients with primary pulmonary arterial hypertension $^{9,16,17}$ and, as such, either drug may also be useful in treating or reducing HPV and/or HAPE. ${ }^{11,18-21}$

Several altitude field studies have reported improved exercise performance with oral administration of sildenafil in healthy humans at high altitude and have shown improved blood oxygen saturation measured via pulse oximetry (Table 1). ${ }^{22-24}$ However, to date, only Richalet et $\mathrm{al}^{23}$ have reported arterial $\mathrm{PO}_{2}\left(\mathrm{PaO}_{2}\right)$ measurements during exercise at real altitude and, here, $\mathrm{PaO}_{2}$ is only reported at ventilatory threshold after 2 and 5 days at high altitude following some acclimitization. Since it is well-established that pulmonary gas exchange is impaired during exercise under acute hypoxic conditions-manifesting as a reduced $\mathrm{PaO}_{2}$ and a widening $\mathrm{A}-\mathrm{aDO} 2-$ we sought to determine whether taking either sildenafil or bosentan would improve arterial oxygenation and pulmonary gas exchange during acute exposure to hypoxia. We hypothesized that pulmonary vasodilation, via sildenafil or bosentan, would improve pulmonary gas exchange during acute hypoxic exercise, resulting in a greater $\mathrm{PaO}_{2}$ and $\mathrm{SaO}_{2}$ and a reduced $\mathrm{A}-\mathrm{aDO}_{2}$ compared to the same level of exercise in hypoxia without either drug. Furthermore, because of the burgeoning interest in gender-based differences in gas exchange during exercise, ${ }^{25-28}$ we also compared the drug treatment responses between our male and female subjects.

\section{Methods}

\section{SUBJECTS}

This study was approved by the Human Subjects Ethics Committee at Innsbruck Medical School and conducted in accordance with guidelines outlined in the Declaration of Helsinki.

A total of 16 subjects ( $n=8$ males, $n=8$ females) were recruited and provided informed consent prior to participating in a single-blinded repeated-measure (no-drug/ drug) exercise study. Young athletic university students with no significant medical history were randomly allocated to either the sildenafil (total $\mathrm{N}=10 ; \mathrm{n}=5$ males, $\mathrm{n}=5$ females) or bosentan (total $\mathrm{N}=6 ; n=3$ males, $n=3$ females) group.

\section{INITIAL SCREENING AND MAXIMAL EXERCISE TESTING}

Subjects provided a brief medical history and were screened for cardiovascular (3-lead ECG) and pulmonary (spirometry) disease prior to testing. Each subject performed 2 exercise tests measuring maximal oxygen uptake $\left(\dot{\mathrm{V}}_{2} \max \right)$, one in normoxia and one in hypoxia (fraction of inspired oxygen $\left[\mathrm{FIO}_{2}\right]=0.11 \%$ ) while 


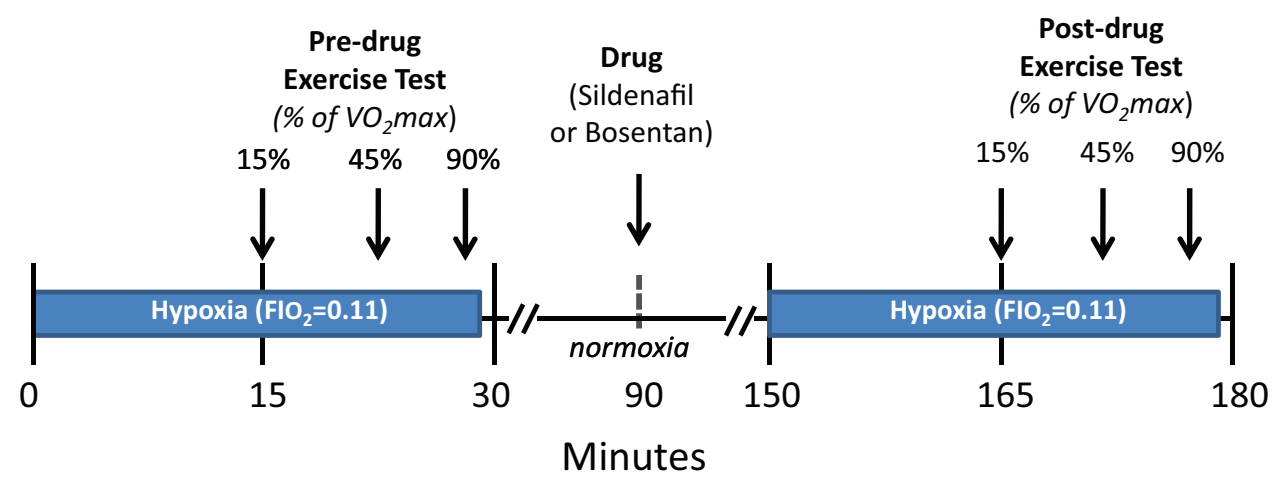

Figure. Timeline depicting the exercise protocol before and after drug treatment.

riding an electronically braked cycle ergometer (Ergometrics 900, Ergoline, Germany). Tests were performed on separate days (no more than 2 days apart) and all hypoxic testing was performed in a commercially available normobaric hypoxic chamber (Hypoxico Inc, New York, NY, USA). Subjects were outfitted with a face mask; measurements of minute ventilation ( $\dot{\mathrm{VE}}), \mathrm{O}_{2}$ consumption $\left(\dot{\mathrm{VO}}_{2}\right), \mathrm{CO}_{2}$ production $\left(\dot{\mathrm{V}}_{2}\right)$, and respiratory exchange ratio (RER) were taken and calculated using Jaeger Oxycon Mobile (Viasys Healthcare Inc., Jaeger, Hoechberg, Germany) equipped with wireless telemetry to a desktop computer. Initial workload for each maximal exercise test began at 50 watts ( 25 watts for females) and was increased by 25 watts at 1 minute intervals until volitional fatigue occurred. Maximal oxygen uptake was defined as the highest $\dot{\mathrm{VO}}_{2}$ achieved during the final workload and it was used to establish the submaximal workload for steady-state exercise before and after drug treatment.

\section{DRUG TREATMENT AND EXERCISE PROTOCOL}

Subjects returned to the laboratory within 1 week of the maximal exercise testing to perform submaximal pre-/ post-drug exercise at light, moderate, and heavy work intensities (which corresponded to $15 \%, 45 \%$, and up to $90 \%$ of $\left.\dot{\mathrm{VO}}_{2} \max \right)$ in hypoxia $\left(\mathrm{FIO}_{2}=0.11\right)$ while arterial blood gases and metabolic measurements $\left(\mathrm{V}_{2}\right.$, $\dot{\mathrm{V} C O}{ }_{2}, \mathrm{RER}$ and $\left.\dot{\mathrm{V}} \mathrm{E}\right)$ were obtained (Figure). Subjects breathed through a face mask fitted to a non-rebreathing valve (Hans-Rudolph 2700, Kansas City, MO, USA) whose expiratory end was connected to a heated mixing box. Each exercise workload was maintained for approximately 5 minutes, with the exception of the last workload, which only lasted about 3 to 4 minutes (based on the subject's ability to complete this near-maximal workload). Blood samples were collected in duplicate at rest and during the last 1 to 2 minutes in each exercise workload. The same exercise was performed before and 1-hour after taking a single oral dose of either sildenafil citrate $(50 \mathrm{mg}$ tablet) or bosentan $(62.5 \mathrm{mg}$ tablet) on the same day (Figure). Subjects were only admitted into one treatment group and were not informed which drug they were given. They were required to rest for at least 2 hours (in room air) between the 2 identical hypoxic exercise tests (ie, pre-drug and post-drug). Ingestion of the drug occurred 1 hour prior to the second exercise bout (Figure).

\section{ARTERIAL SAMPLING AND BLOOD GAS MEASUREMENTS}

Arterial blood was sampled at rest and during exercise from a 20-gauge catheter (BD Arterial Cannula with FloSwitch, Becton-Dickinson, Swindon, UK) inserted in the radial artery. Two $\mathrm{mL}$ of arterial blood was drawn in a separate heparinized syringe and used for arterial blood gas assessment. Arterial blood gas samples were analyzed for partial pressure of $\mathrm{O}_{2}\left(\mathrm{PaO}_{2}\right)$ and $\mathrm{CO}_{2}\left(\mathrm{PaCO}_{2}\right)$, $\mathrm{pH}$, hemoglobin $(\mathrm{Hgb}), \mathrm{O}_{2}$ saturation $\left(\mathrm{SaO}_{2}\right)$, electrolytes, and lactate, using a Bayer Rapidlab 865 blood gas analyzer/co-oximeter (Chiron Diagnostics Corp., Walpole, MA, USA). All blood gas values were corrected for temperature using tympanic temperatures measured during blood sampling.

Alveolar $\mathrm{PO}_{2}$ was calculated using the equation $\mathrm{PAO} 2=$ $\mathrm{PIO} 2$ - $(\mathrm{PaCO} 2 / \mathrm{RER})(1-\mathrm{FIO} 2$ [1-RER] $)$ and was used to calculate alveolar minus arterial $\mathrm{O}_{2}$ difference $\left(\mathrm{A}-\mathrm{aDO}_{2}\right)$.

\section{STATISTICAL ANALYSES}

Data presented are expressed as mean \pm SE, unless otherwise noted. Statistical analysis was first performed using a $3 \times 2$ analysis of variance (ANOVA) - drug (sildenafil vs bosentan) before and after treatment (with vs without drug) and exercise intensity (StatView 5.0, SAS Institute, Inc, Cary, NC, USA). When a significant main effect for the drug was found, separate post-hoc analysis (Boneferroni-Dunn) was performed on sildena- 
Table 2. Comparison of subject's pulmonary function and maximal aerobic capacity based on separate grouping for gender and drug treatment

\begin{tabular}{|c|c|c|c|c|}
\hline & \multicolumn{2}{|c|}{ Gender } & \multicolumn{2}{|c|}{ Drug Treatment } \\
\hline & Male & Female & Sildenafil & Bosentan \\
\hline Number of subjects $(\mathrm{m} / \mathrm{f})$ & 8 & 8 & $10(5 / 5)$ & $6(3 / 3)$ \\
\hline Age (years) & $27 \pm 6$ & $26 \pm 3$ & $25 \pm 3$ & $28 \pm 7$ \\
\hline Height $(\mathrm{cm})$ & $180 \pm 5$ & $169 \pm 5^{*}$ & $175 \pm 7$ & $175 \pm 8$ \\
\hline Body Mass (kg) & $70 \pm 5$ & $59 \pm 5^{*}$ & $64 \pm 8$ & $65 \pm 7$ \\
\hline $\mathrm{FVC}(\mathrm{L})$ & $5.63 \pm 0.47$ & $4.35 \pm 0.67 *$ & $4.94 \pm 1.01$ & $5.17 \pm 0.2$ \\
\hline FEV1 (L) & $4.83 \pm 0.23$ & $3.78 \pm .45^{*}$ & $4.27 \pm 0.78$ & $4.49 \pm 0.31$ \\
\hline FEV1/FVC (\%) & $86 \pm 6$ & $87 \pm 4$ & $87 \pm 5$ & $87 \pm 5$ \\
\hline FVC (\% pred) & $101 \pm 8$ & $106 \pm 17$ & $100 \pm 9$ & $109 \pm 17$ \\
\hline FEV1 (\% pred) & $105 \pm 6$ & $107 \pm 12$ & $103 \pm 8$ & $111 \pm 9$ \\
\hline FEV1/FVC (\% pred) & $104 \pm 7$ & $102 \pm 5$ & $103 \pm 6$ & $103 \pm 8$ \\
\hline \multicolumn{5}{|l|}{ Normoxia } \\
\hline $\mathrm{VO}_{2} \max (\mathrm{L} / \mathrm{min})$ & $3.90 \pm 0.35$ & $2.78 \pm 0.29 *$ & $3.25 \pm 0.75$ & $3.48 \pm 0.5$ \\
\hline $\mathrm{VO}_{2} \max (\mathrm{mL} / \mathrm{kg} / \mathrm{min})$ & $55.9 \pm 6.3$ & $47.3 \pm 4.5^{*}$ & $50.6 \pm 8.2$ & $53.9 \pm 4.1$ \\
\hline \multicolumn{5}{|l|}{ Hypoxia } \\
\hline $\mathrm{VO}_{2} \max (\mathrm{L} / \mathrm{min})$ & $3.02 \pm 0.20$ & $2.36 \pm 0.37 *$ & $2.61 \pm 0.51$ & $2.82 \pm 0.30$ \\
\hline $\mathrm{VO}_{2} \max (\mathrm{mL} / \mathrm{kg} / \mathrm{min})$ & $43.2 \pm 4.1$ & $37.8 \pm 3.1 *$ & $40.3 \pm 4.1$ & $42 \pm 4.6$ \\
\hline
\end{tabular}

Data are mean $\pm \mathrm{SD}$. FVC, forced vital capacity; FEV1, forced expiratory volume at $1 \mathrm{sec} ; \mathrm{VO}_{2}$ max, maximal $\mathrm{O}_{2}$ uptake. $* \mathrm{p}<.05$ using unpaired Student's $t$-test.

fil and bosentan groups. When a significant main effect for the drug treatment was found, repeated measures ANOVA was used for determination of significant differences before and after drug treatment. Anthropometric data between groups was compared using an unpaired $t$ test analysis. In all cases, significance was determined by a $P$ value $<.05$.

\section{Results}

\section{SUBJECT CHARACTERISTICS AND PRE-SCREENING DATA}

Subject demographics, pulmonary function data, and maximal oxygen uptake $\left(\dot{\mathrm{VO}}_{2} \max \right)$ are shown in Table 2. There was no significant difference in age among the subjects based on grouping by gender or drug treatment. As expected, females had lower body mass, shorter stature, smaller lung volumes, and lower $\dot{\mathrm{V}}_{2}$ max compared to male counterparts (Table 2). However, when subjects were grouped according to drug treatment, no significant differences in body mass, pulmonary function, and/or $\dot{\mathrm{VO}}_{2} \mathrm{max}$ existed. Regardless of grouping, the data in Table 2 indicate that all subjects had normal pulmonary function and represent a very aerobically fit population.

\section{METABOLIC AND BLOOD GAS DATA}

As expected, $\dot{\mathrm{VO}}_{2}, \dot{\mathrm{V}} \mathrm{CO}_{2}$, and $\dot{\mathrm{V} E}$ during submaximal exercise were lower in women compared to men (Table 3), but no difference was seen in $\dot{\mathrm{V}} \mathrm{O}_{2}, \dot{\mathrm{V} C O}{ }_{2}$, and $\dot{\mathrm{VE}}$ in response to either sildenafil or bosentan drug treatment (Table 4). The RER, at rest or during exercise, was unaffected by either gender or drug treatment (Tables 3 and 4).

Treatment with either sildenafil or bosentan increased $\mathrm{PaO}_{2}$ by 2 to $3 \mathrm{~mm} \mathrm{Hg}$ and $\mathrm{SaO}_{2}$ by $3 \%$ to $4 \%$ (Table 4) with similar increases observed in both male and females (Table 3). Drug treatment, however, had no significant effect on either $\mathrm{A}-\mathrm{aDO}_{2}$ and $\mathrm{P}_{50}$ (ie, $\mathrm{PaO}_{2}$ at $50 \%$ saturation, Table 4) except for a small increase in $\mathrm{A}-\mathrm{aDO}_{2}$ seen in females (Table 3). However, $\mathrm{PaCO}_{2}$ was found to be lower $(P<.001)$ with sildenafil, but not significantly different with bosentan.

Significant main effect for gender was observed with arterial $\mathrm{PO}_{2}$ and saturation $\left(\mathrm{SaO}_{2}\right)$, indicating that females had higher $\mathrm{PaO}_{2}$ and $\mathrm{SaO}_{2}$ compared to men at any given exercise level (Table 3). However, no significant main effect in $\mathrm{A}-\mathrm{aDO}_{2}$ and $\mathrm{P}_{50}$ was observed between the genders (Table 3). Likewise, arterial $\mathrm{pH}$ was not different based on gender or drug treatment grouping (Tables 3 and 4).

\section{CARDIAC DATA}

Heart rate, at rest and during exercise, was not different between males and females (Table 3). However, following treatment with either sildenafil or bosentan, heart rate was greater compared to pre-drug measurements made at rest and during exercise in both males and females (Tables 3 and 4). 
Table 3. Metabolic and blood gas data in hypoxia $\left(\mathrm{FIO}_{2}=0.11\right)$ at rest and during exercise before and after drug (sildenafil or bosentan) treatment

\begin{tabular}{|c|c|c|c|c|c|c|c|}
\hline & \multicolumn{2}{|c|}{ Male $(n=8)$} & \multirow[b]{2}{*}{ Drug Effect } & \multicolumn{2}{|c|}{ Female $(n=8)$} & \multirow[b]{2}{*}{ Drug Effect } & \multirow{2}{*}{$\begin{array}{c}\text { Gender } \\
\text { Effect }\end{array}$} \\
\hline & Before Drug & After Drug & & Before Drug & After Drug & & \\
\hline \multicolumn{8}{|l|}{ Watts } \\
\hline Rest & $0 \pm 0$ & $0 \pm 0$ & \multirow[t]{4}{*}{ n.s. } & $0 \pm 0$ & $0 \pm 0$ & \multirow[t]{4}{*}{ n.s. } & \multirow[t]{4}{*}{$<0.001$} \\
\hline Light & $53 \pm 3$ & $53 \pm 3$ & & $28 \pm 3$ & $28 \pm 3$ & & \\
\hline Moderate & $131 \pm 6$ & $131 \pm 6$ & & $88 \pm 5$ & $88 \pm 5$ & & \\
\hline Heavy & $203 \pm 9$ & $203 \pm 9$ & & $150 \pm 5$ & $150 \pm 5$ & & \\
\hline \multicolumn{8}{|c|}{ Heart rate (bpm) } \\
\hline Rest & $86 \pm 4$ & $99 \pm 7$ & \multirow{4}{*}{$<0.01$} & $91 \pm 5$ & $102 \pm 5$ & \multirow[t]{4}{*}{$<0.01$} & \multirow[t]{4}{*}{ n.s. } \\
\hline Light & $123 \pm 6$ & $130 \pm 7$ & & $113 \pm 6$ & $127 \pm 5$ & & \\
\hline Moderate & $152 \pm 7$ & $159 \pm 5$ & & $150 \pm 4$ & $157 \pm 2$ & & \\
\hline Heavy & $173 \pm 4$ & $177 \pm 4$ & & $173 \pm 4$ & $175 \pm 3$ & & \\
\hline \multicolumn{8}{|c|}{$\mathrm{V}_{\mathrm{E}}(\mathrm{L} / \mathrm{min}, \mathrm{BTPS})$} \\
\hline Rest & $21 \pm 2$ & $21 \pm 2$ & \multirow[t]{4}{*}{ n.s. } & $17 \pm 2$ & $18 \pm 1$ & \multirow[t]{4}{*}{ n.s. } & \multirow[t]{4}{*}{0.01} \\
\hline Light & $42 \pm 3$ & $43 \pm 2$ & & $28 \pm 2 *$ & $32 \pm 2 *$ & & \\
\hline Moderate & $77 \pm 6$ & $77 \pm 6$ & & $54 \pm 4^{*}$ & $57 \pm 4^{*}$ & & \\
\hline Heavy & $114 \pm 5$ & $117 \pm 5$ & & $86 \pm 4 *$ & $91 \pm 5^{*}$ & & \\
\hline \multicolumn{8}{|c|}{$\mathrm{VO}_{2}(\mathrm{~L} / \mathrm{min}, \mathrm{STPD})$} \\
\hline Rest & $0.60 \pm 0.06$ & $0.59 \pm 0.06$ & \multirow[t]{4}{*}{ n.s. } & $0.49 \pm 0.06$ & $0.50 \pm 0.05$ & \multirow[t]{4}{*}{ n.s. } & $<0.001$ \\
\hline Light & $1.45 \pm 0.06$ & $1.42 \pm 0.07$ & & $0.91 \pm 0.06^{*}$ & $0.96 \pm 0.05^{*}$ & & \\
\hline Moderate & $2.24 \pm 0.08$ & $2.26 \pm 0.09$ & & $1.58 \pm 0.09 *$ & $1.61 \pm 0.11 *$ & & \\
\hline Heavy & $2.86 \pm 0.06$ & $2.86 \pm 0.05$ & & $2.11 \pm 0.10^{*}$ & $2.13 \pm 0.10 *$ & & \\
\hline $\begin{array}{c}\mathrm{VCO}_{2}(\mathrm{~L} / \mathrm{mi} \\
\mathrm{STPD})\end{array}$ & & & & & & & \\
\hline Rest & $0.45 \pm 0.03$ & $0.45 \pm 0.03$ & n.s. & $0.37 \pm 0.04$ & $0.39 \pm 0.03$ & n.s. & $<0.001$ \\
\hline Light & $1.12 \pm 0.06$ & $1.12 \pm 0.07$ & & $0.71 \pm 0.04 *$ & $0.74 \pm 0.04 *$ & & \\
\hline Moderate & $2.01 \pm 0.16$ & $1.96 \pm 0.10$ & & $1.35 \pm 0.06^{*}$ & $1.40 \pm 0.09 *$ & & \\
\hline Heavy & $2.84 \pm 0.17$ & $2.90 \pm 0.17$ & & $2.11 \pm 0.05^{*}$ & $2.17 \pm 0.07 *$ & & \\
\hline RER & & & & & & & \\
\hline Rest & $0.76 \pm 0.03$ & $0.79 \pm 0.04$ & n.s. & $0.76 \pm 0.04$ & $0.79 \pm 0.04$ & n.s. & n.s. \\
\hline Light & $0.78 \pm 0.03$ & $0.80 \pm 0.05$ & & $0.79 \pm 0.04$ & $0.78 \pm 0.05$ & & \\
\hline Moderate & $0.90 \pm 0.07$ & $0.88 \pm 0.05$ & & $0.87 \pm 0.04$ & $0.89 \pm 0.05$ & & \\
\hline Heavy & $1.00 \pm 0.07$ & $1.02 \pm 0.07$ & & $1.02 \pm 0.07$ & $1.03 \pm 0.04$ & & \\
\hline $\mathrm{pHa}$ & & & & & & & \\
\hline Rest & $7.46 \pm 0.00$ & $7.45 \pm 0.01$ & n.s. & $7.44 \pm 0.01$ & $7.44 \pm 0.01$ & n.s. & n.s. \\
\hline Light & $7.44 \pm 0.01$ & $7.44 \pm 0.01$ & & $7.43 \pm 0.01$ & $7.43 \pm 0.01$ & & \\
\hline Moderate & $7.40 \pm 0.01$ & $7.41 \pm 0.01$ & & $7.40 \pm 0.01$ & $7.40 \pm 0.01$ & & \\
\hline Heavy & $7.33 \pm 0.02$ & $7.33 \pm 0.02$ & & $7.33 \pm 0.01$ & $7.34 \pm 0.01$ & & \\
\hline $\mathrm{PaCO}_{2}(\mathrm{mml}$ & & & & & & & \\
\hline Rest & $33.8 \pm 0.5$ & $32.1 \pm 0.7$ & n.s. & $33.3 \pm 0.5$ & $30.9 \pm 0.6$ & n.s. & n.s. \\
\hline Light & $34.4 \pm 0.5$ & $32.2 \pm 0.5$ & & $33.9 \pm 0.6$ & $30.6 \pm 1.0$ & & \\
\hline Moderate & $33.5 \pm 0.6$ & $30.6 \pm 1.9$ & & $33.6 \pm 0.9$ & $30.2 \pm 1.2$ & & \\
\hline Heavy & $31.9 \pm 0.4$ & $31.6 \pm 1.5$ & & $32.5 \pm 1.0$ & $30.1 \pm 1.2$ & & \\
\hline $\mathrm{PaO}_{2}(\mathrm{mmH}$ & & & & & & & \\
\hline Rest & $37.9 \pm 1.0$ & $40.8 \pm 0.9$ & $<0.001$ & $41.3 \pm 1.1^{*}$ & $44.0 \pm 0.8^{*}$ & 0.002 & 0.002 \\
\hline Light & $33.4 \pm 0.9$ & $35.8 \pm 0.4$ & & $38.6 \pm 0.7 *$ & $40.3 \pm 0.7 *$ & & \\
\hline Moderate & $32.8 \pm 0.9$ & $35.0 \pm 0.4$ & & $37.7 \pm 0.5^{*}$ & $38.9 \pm 0.6^{*}$ & & \\
\hline Heavy & $34.8 \pm 0.7$ & $36.6 \pm 0.7$ & & $38.6 \pm 0.8^{*}$ & $40.2 \pm 0.9 *$ & & \\
\hline $\mathrm{SaO}_{2}(\%)$ & & & & & & & \\
\hline Rest & $77 \pm 1$ & $80 \pm 1$ & $<0.001$ & $79 \pm 1$ & $83 \pm 1 *$ & $<0.001$ & 0.01 \\
\hline Light & $68 \pm 2$ & $72 \pm 1$ & & $74 \pm 1^{*}$ & $78 \pm 1^{*}$ & & \\
\hline
\end{tabular}


Table 3. Continued

\begin{tabular}{|c|c|c|c|c|c|c|c|}
\hline & \multicolumn{2}{|c|}{ Male $(n=8)$} & \multirow[b]{2}{*}{ Drug Effect } & \multicolumn{2}{|c|}{ Female $(n=8)$} & \multirow[b]{2}{*}{ Drug Effect } & \multirow{2}{*}{$\begin{array}{l}\text { Gender } \\
\text { Effect }\end{array}$} \\
\hline & Before Drug & After Drug & & Before Drug & After Drug & & \\
\hline Moderate & $64 \pm 2$ & $68 \pm 1$ & $71 \pm 1^{*}$ & $74 \pm 1^{*}$ & & & \\
\hline Heavy & $61 \pm 1$ & $65 \pm 1$ & $66 \pm 2 *$ & $70 \pm 1^{*}$ & & & \\
\hline \multicolumn{8}{|c|}{$\mathrm{A}-\mathrm{aDO}_{2}(\mathrm{mmHg})$} \\
\hline Rest & $-4 \pm 2$ & $-3 \pm 2$ & n.s. & $-6 \pm 2$ & $-5 \pm 1$ & 0.034 & n.s. \\
\hline Light & $-1 \pm 2$ & $2 \pm 2$ & & $-3 \pm 1$ & $-1 \pm 1$ & & \\
\hline Moderate & $5 \pm 2$ & $8 \pm 3$ & & $2 \pm 1$ & $5 \pm 2$ & & \\
\hline Heavy & $9 \pm 2$ & $10 \pm 2$ & & $7 \pm 1$ & $9 \pm 1$ & & \\
\hline $\mathrm{P}_{50}$ & $26 \pm 1$ & $23 \pm 1$ & n.s. & $23 \pm 1$ & $21 \pm 1$ & 0.048 & n.s. \\
\hline
\end{tabular}

Data are mean \pm SE. $*$ significantly different compared to males in same group, $\mathrm{p}<.05$

Systolic, diastolic, and mean arterial pressure were not different, at rest or during exercise, when comparing gender (data not shown). However, systolic pressure was $10 \%$ lower with sildenafil $(P<.05)$, but not significantly different with bosentan $(P=.32)$, despite a similar $9 \%$ decline at rest; and no difference in systolic pressure was observed with either sildenafil or bosentan during heavy exercise. Likewise, no differences in diastolic or mean arterial pressure were seen at rest or during heavy exercise with either drug (data not shown).

\section{Discussion}

The main finding of this study is that, in a controlled laboratory trial, sildenafil and bosentan significantly increased arterial oxygenation (ie, $\mathrm{PaO}_{2}$ and $\mathrm{SaO}_{2}$ ) at rest and during exercise during short-term exposure to acute hypoxia. We also examined whether taking either drug would be influenced by gender. In this regard, there was no systematic difference between the drug-treated men and women in hypoxia, at rest or during exercise. However, these data do show that women (with or without drug treatment) were less hypoxemic at rest and during exercise in hypoxia compared to their male counterparts.

\section{IMPROVED PULMONARY GAS EXCHANGE IN HYPOXIA WITH SILDENAFIL OR BOSENTAN TREATMENT}

In healthy individuals, pulmonary $\mathrm{O}_{2}$ transport is normally perfusion-limited and, thus, arterial oxygenation is largely dependent on blood flow through the lungs. However, under hypoxic conditions (especially during exercise), $\mathrm{O}_{2}$ can become diffusion-limited (impairing $\mathrm{O}_{2}$ transfer across the alveolar-capillary membrane), resulting in a widening $\mathrm{A}-\mathrm{aDO}$. By definition, the diffusing capacity of $\mathrm{O}_{2}$ in the lung $\left(\mathrm{DL}_{\mathrm{O} 2}\right)$ is determined by 3 components: 1) the alveolar-capillary membrane $\left(D_{M}\right)$; 2) the gas $\left(\mathrm{O}_{2}\right)$ pressure differential $(\Delta \mathrm{P})$ across $\mathrm{D}_{\mathrm{M}}$; and 3) the time it takes for $\mathrm{O}_{2}$ to react with hemoglobin (which is a function of $\mathrm{O}_{2}$ reaction time $[\theta]$ and the volume of capillary blood $\left.\left[\mathrm{V}_{\mathrm{c}}\right]\right)$. Thus, increasing pulmonary blood flow (by reducing PVR) would not be expected to benefit an $\mathrm{O}_{2}$ diffusion-limited lung. However, Snyder et al have shown an improved lung $\mathrm{D}_{\mathrm{M}}$ conductance when correcting for the changes in pulmonary $\mathrm{V}_{\mathrm{c}}$ following sildenafil treatment. ${ }^{29}$ These data suggest that, while reducing PVR may not alter pulmonary blood flow per se (ie, cardiac output), ${ }^{15}$ sildenafil may increase pulmonary capillary volume and lessen the effects of the $\mathrm{O}_{2}$ diffusion-limitation under hypoxic conditions by decreasing capillary transit time within the pulmonary capillaries. $^{29}$

Other potential explanations of improved gas exchange that must be considered include: 1) improved ventilation/perfusion matching ( $\dot{\mathrm{V} A} / \dot{\mathrm{Q}}) ; 2$ ) reduced shunting; and/or 3) hyperventilation. From these data, it cannot be directly addressed whether or not $\dot{\mathrm{V} A} / \dot{\mathrm{Q}}$ was improved or if a reduction in pulmonary shunting also occurred. But, we would note that, in mechanically ventilated pigs under normoxic conditions, VंA/Q was not significantly altered with sildenafil dosages at 25,50 , or $100 \mathrm{mg} .{ }^{30}$ It is not known whether the same is true under hypoxic conditions in the face of HPV. To the extent that HPV is unevenly distributed in healthy human lungs, it is possible that converting low $\dot{\mathrm{V} A} / \dot{Q}$ lung regions to normal-or more homogeneous- $\dot{V} A / \dot{Q}$ units could improve gas exchange and arterial oxygenation. Indeed, because impaired pulmonary gas exchange and widening $\mathrm{A}-\mathrm{aDO}_{2}$ are thought to be linked with the development of pulmonary hypertension (leading to pulmonary interstitial fluid accumulation) and VA/Q mismatching with exercise at high altitude, ${ }^{1,3}$ it is not surprising that reducing pul- 
Table 4. Metabolic and blood gas data in hypoxia $\left(\mathrm{FIO}_{2}=0.11\right)$ at rest and during exercise before and after drug treatment

\begin{tabular}{|c|c|c|c|c|c|c|c|}
\hline & \multicolumn{2}{|c|}{ Sildenafil $(n=10)$} & \multirow[b]{2}{*}{ Drug Effect } & \multicolumn{2}{|c|}{ Bosentan $(n=6)$} & \multirow[b]{2}{*}{$\begin{array}{l}\text { Drug } \\
\text { Effect }\end{array}$} & \multirow[b]{2}{*}{$\begin{array}{c}\text { Sil vs Bos } \\
\text { Effect }\end{array}$} \\
\hline & $\begin{array}{c}\text { Before } \\
\text { Drug }\end{array}$ & After Drug & & $\begin{array}{c}\text { Before } \\
\text { Drug }\end{array}$ & After Drug & & \\
\hline \multicolumn{8}{|l|}{ Watts } \\
\hline Rest & $0 \pm 0$ & $0 \pm 0$ & \multirow[t]{4}{*}{ n.s. } & $0 \pm 0$ & $0 \pm 0$ & \multirow[t]{4}{*}{ n.s. } & \multirow[t]{4}{*}{ n.s. } \\
\hline Light & $40 \pm 4$ & $40 \pm 4$ & & $38 \pm 6$ & $38 \pm 6$ & & \\
\hline Moderate & $113 \pm 10$ & $113 \pm 10$ & & $113 \pm 6$ & $113 \pm 6$ & & \\
\hline Heavy & $185 \pm 12$ & $185 \pm 12$ & & $171 \pm 10$ & $171 \pm 10$ & & \\
\hline \multicolumn{8}{|c|}{ Heart rate (bpm) } \\
\hline Rest & $86 \pm 4$ & $102 \pm 6$ & \multirow{4}{*}{$<0.01$} & $92 \pm 6$ & $98 \pm 6$ & \multirow[t]{4}{*}{$<0.01$} & \multirow[t]{4}{*}{ n.s. } \\
\hline Light & $121 \pm 6$ & $132 \pm 6$ & & $114 \pm 6$ & $121 \pm 4$ & & \\
\hline Moderate & $153 \pm 5$ & $161 \pm 4$ & & $147 \pm 6$ & $152 \pm 2$ & & \\
\hline Heavy & $174 \pm 12$ & $177 \pm 3$ & & $171 \pm 4$ & $175 \pm 5$ & & \\
\hline \multicolumn{8}{|c|}{$\mathrm{V}_{\mathrm{E}}(\mathrm{L} / \mathrm{min}, \mathrm{BTPS})$} \\
\hline Rest & $15 \pm 1$ & $16 \pm 1$ & \multirow{4}{*}{$\begin{array}{l}n . s . \\
(0.07)\end{array}$} & $23 \pm 2$ & $22 \pm 2$ & \multirow[t]{4}{*}{ n.s. } & \multirow[t]{4}{*}{ n.s. } \\
\hline Light & $34 \pm 3$ & $36 \pm 2$ & & $35 \pm 4$ & $36 \pm 3$ & & \\
\hline Moderate & $58 \pm 5$ & $61 \pm 5$ & & $69 \pm 5$ & $69 \pm 4$ & & \\
\hline Heavy & $95 \pm 6$ & $100 \pm 6$ & & $102 \pm 7$ & $103 \pm 5$ & & \\
\hline \multicolumn{8}{|c|}{$\mathrm{VO}_{2}(\mathrm{~L} / \mathrm{min}, \mathrm{STPD})$} \\
\hline Rest & $0.42 \pm 0.03$ & $0.44 \pm 0.02$ & \multirow[t]{4}{*}{ n.s. } & $0.54 \pm 0.05$ & $0.52 \pm 0.06$ & n.s. & n.s. \\
\hline Light & $1.10 \pm 0.10$ & $1.11 \pm 0.08$ & & $0.98 \pm 0.13$ & $0.99 \pm 0.12$ & & \\
\hline Moderate & $1.71 \pm 0.13$ & $1.72 \pm 0.13$ & & $1.73 \pm 0.15$ & $1.76 \pm 0.14$ & & \\
\hline Heavy & $2.34 \pm 0.17$ & $2.34 \pm 0.16$ & & $2.26 \pm 0.19$ & $2.24 \pm 0.15$ & & \\
\hline $\mathrm{VCO}_{2}(\mathrm{~L} / \mathrm{mir}$ & & & & & & & \\
\hline Rest & $0.34 \pm 0.03$ & $0.37 \pm 0.02$ & n.s. & $0.46 \pm 0.03$ & $0.45 \pm 0.05$ & n.s. & n.s. \\
\hline Light & $0.92 \pm 0.09$ & $0.98 \pm 0.09$ & & $0.82 \pm 0.10$ & $0.82 \pm 0.09$ & & \\
\hline Moderate & $1.59 \pm 0.13$ & $1.68 \pm 0.14$ & & $1.67 \pm 0.10$ & $1.64 \pm 0.09$ & & \\
\hline Heavy & $2.51 \pm 0.18$ & $2.57 \pm 0.18$ & & $2.48 \pm 0.16$ & $2.52 \pm 0.18$ & & \\
\hline RER & & & & & & & \\
\hline Rest & $0.81 \pm 0.03$ & $0.84 \pm 0.03$ & n.s. & $0.86 \pm 0.03$ & $0.83 \pm 0.03$ & n.s. & n.s. \\
\hline Light & $0.84 \pm 0.03$ & $0.88 \pm 0.03$ & & $0.84 \pm 0.03$ & $0.87 \pm 0.03$ & & \\
\hline Moderate & $0.93 \pm 0.04$ & $0.97 \pm 0.04$ & & $0.96 \pm 0.04$ & $0.97 \pm 0.03$ & & \\
\hline Heavy & $1.09 \pm 0.06$ & $1.10 \pm 0.03$ & & $1.11 \pm 0.05$ & $1.11 \pm 0.03$ & & \\
\hline $\mathrm{pHa}$ & & & & & & & \\
\hline Rest & $7.45 \pm 0.01$ & $7.44 \pm 0.01$ & n.s. & $7.45 \pm 0.01$ & $7.45 \pm 0.01$ & n.s. & n.s. \\
\hline Light & $7.43 \pm 0.01$ & $7.43 \pm 0.01$ & & $7.44 \pm 0.01$ & $7.44 \pm 0.01$ & & \\
\hline Moderate & $7.40 \pm 0.01$ & $7.41 \pm 0.01$ & & $7.40 \pm 0.01$ & $7.41 \pm 0.01$ & & \\
\hline Heavy & $7.33 \pm 0.01$ & $7.34 \pm 0.02$ & & $7.33 \pm 0.02$ & $7.33 \pm 0.02$ & & \\
\hline $\mathrm{PaCO}_{2}(\mathrm{~mm}$ & & & & & & & \\
\hline Rest & $33.9 \pm 0.3$ & $31.6 \pm 0.5$ & $<0.001$ & $32.9 \pm 0.8$ & $31.4 \pm 1.0$ & n.s. & n.s. \\
\hline Light & $34.5 \pm 0.5$ & $31.3 \pm 0.8$ & & $33.6 \pm 0.6$ & $31.6 \pm 1.0$ & & \\
\hline Moderate & $34.2 \pm 0.6$ & $30.2 \pm 1.2$ & & $32.4 \pm 0.8$ & $30.7 \pm 2.2$ & & \\
\hline Heavy & $32.8 \pm 0.5$ & $30.6 \pm 0.9$ & & $31.1 \pm 0.9$ & $31.2 \pm 2.3$ & & \\
\hline $\mathrm{PaO}_{2}(\mathrm{mmHg}$ & & & & & & & \\
\hline Rest & $39.6 \pm 1.3$ & $42.0 \pm 1.0$ & 0.01 & $39.6 \pm 0.8$ & $43.1 \pm 0.8$ & 0.02 & n.s. \\
\hline Light & $35.9 \pm 1.2$ & $38.2 \pm 1.0$ & & $36.2 \pm 1.2$ & $37.8 \pm 1.1$ & & \\
\hline Moderate & $35.1 \pm 1.1$ & $36.8 \pm 0.8$ & & $35.6 \pm 1.2$ & $37.2 \pm 1.1$ & & \\
\hline Heavy & $36.1 \pm 1.0$ & $38.0 \pm 0.9$ & & $37.7 \pm 1.1$ & $39.0 \pm 1.1$ & & \\
\hline $\mathrm{SaO}_{2}(\%)$ & & & & & & & \\
\hline Rest & $78 \pm 1$ & $81 \pm 1$ & 0.001 & $78 \pm 1$ & $82 \pm 1$ & 0.01 & n.s. \\
\hline Light & $71 \pm 2$ & $75 \pm 1$ & & $71 \pm 2$ & $75 \pm 2$ & & \\
\hline Moderate & $67 \pm 2$ & $71 \pm 1$ & & $68 \pm 2$ & $71 \pm 2$ & & \\
\hline Heavy & $63 \pm 2$ & $67 \pm 1$ & & $65 \pm 2$ & $68 \pm 2$ & & \\
\hline
\end{tabular}


Table 4. Continued

\begin{tabular}{|c|c|c|c|c|c|c|c|}
\hline & \multicolumn{2}{|c|}{ Sildenafil $(n=10)$} & \multirow[b]{2}{*}{$\begin{array}{l}\text { Drug } \\
\text { Effect }\end{array}$} & \multicolumn{2}{|c|}{ Bosentan $(n=6)$} & \multirow[b]{2}{*}{$\begin{array}{l}\text { Drug } \\
\text { Effect }\end{array}$} & \multirow[b]{2}{*}{$\begin{array}{c}\text { Sil vs Bos } \\
\quad \text { Effect }\end{array}$} \\
\hline & $\begin{array}{c}\text { Before } \\
\text { Drug }\end{array}$ & After Drug & & $\begin{array}{c}\text { Before } \\
\text { Drug }\end{array}$ & After Drug & & \\
\hline \multicolumn{8}{|c|}{$\mathrm{A}-\mathrm{aDO}_{2}(\mathrm{mmHg})$} \\
\hline Rest & $-4 \pm 2$ & $-2 \pm 1$ & n.s. & $0 \pm 2$ & $0 \pm 1$ & n.s. & n.s. \\
\hline Light & $0 \pm 1$ & $3 \pm 1$ & & $2 \pm 1$ & $4 \pm 1$ & & \\
\hline Moderate & $5 \pm 2$ & $9 \pm 2$ & & $9 \pm 1$ & $10 \pm 3$ & & \\
\hline Heavy & $10 \pm 1$ & $11 \pm 1$ & & $12 \pm 1$ & $13 \pm 1$ & & \\
\hline $\mathrm{P}_{50}$ & $25 \pm 1$ & $23 \pm 1$ & 0.041 & $23 \pm 1$ & $21 \pm 2$ & n.s & n.s. \\
\hline
\end{tabular}

Data are mean $\pm \mathrm{SE}$.

monary vascular resistance might increase $\mathrm{PaO}_{2}$ under hypoxic conditions. Supporting this is the observation that HAPE-susceptible subjects develop greater exercise-induced $\dot{V} A / Q$ inequality compared to control subjects, ${ }^{4}$ and that an increase in PAP is thought to be one of the principal mechanisms resulting in HAPE. ${ }^{5-7}$ We cannot exclude the possibility that either alveolar and/or interstitial fluid accumulation (ie, increased leaking of water from the capillaries because of greater capillary permeability, whether as a result of increased PAP or independent of PAP) could itself contribute to diffusion impairment of $\mathrm{O}_{2}$ that is often seen in hypoxic conditions. Nonetheless, the present finding that drugs which reduce PVR result in improved $\mathrm{PaO}_{2}$ during hypoxic exercise is consistent with the notion that HPV and pulmonary hypertension are significant contributors to pulmonary gas exchange impairment, and it also suggests that $\dot{V} A / Q$ was likely altered in order to improve gas exchange following sildenafil or bosentan treatment. Supporting this, a recent study showed that treating patients with COPDassociated pulmonary hypertension with sildenafil worsens VA/Q matching. ${ }^{31}$ Worsening gas exchange (ie, $\dot{V} A / \dot{Q}$ ) could be expected in COPD patients since global vasodilation of the pulmonary vascular bed would work against the beneficial action of HPV. Indeed, in the case of COPD, HPV is needed to redistribute pulmonary blood flow away from damaged (poor gas-exchanging) lung regions and preferentially send blood to areas of the lung with better gas exchange. In contrast, for healthy individuals exposed to environmental hypoxia, it would be postulated that drugs like sildenafil and bosentan would reduce (or prevent) hypoxia-induced pulmonary vasoconstriction and, therefore, mitigate (or prevent) the development of pulmonary hypertension sequelae.

In contrast to $\dot{\mathrm{V}} \mathrm{A} / \mathrm{Q}$, it appears unlikely that hyperventilation played a significant role in improving $\mathrm{PaO}_{2}$.
Although a statistically significant decrease in $\mathrm{PaCO}_{2}$ was observed with sildenafil treatment, this did not translate to a significant elevation in ventilation (Table 4). Moreover, a significant decrease in $\mathrm{PaCO}_{2}$ was not seen with bosentan treatment, which demonstrated similar increases in $\mathrm{PaO}_{2}$ as that seen with sildenafil (Table 4). Thus, it is seems unlikely that hyperventilation can explain the greater $\mathrm{PaO}_{2}$ occurring with sildenafil or bosentan treatment.

It is worth noting that there was a leftward shift in the $\mathrm{O}_{2}$ dissociation curve, as evident by the decline in $\mathrm{P}_{50}$ with sildenafil and a similar decline (albeit nonsignificant) with bosentan treatment (Table 4). Given the hypoxic conditions used in this study $\left(\mathrm{FIO}_{2}=0.11\right)$, it is evident that $\mathrm{PaO}_{2}$ will be positioned on the steep linear portion of the $\mathrm{O}_{2}$ dissociation curve. It is tempting to speculate that, had the $\mathrm{P}_{50}$ not declined, it is possible that a greater post-drug increase in $\mathrm{PaO} 2$ and $\mathrm{SaO} 2$ might have occurred. Nevertheless, with the degree of hypoxemia present, even the relatively small (2-3 Torr) increase in $\mathrm{PaO}_{2}$ we observed could be expected to aid and improve $\mathrm{O}_{2}$ availability to exercising muscles and, consequently, might improve exercise tolerance and/or performance at altitude. ${ }^{18,20,24}$

\section{CARDIAC FUNCTION}

One of the benefits of reducing PVR during exercise in hypoxia may be a reduction in right ventricular afterload-aiding left ventricular diastolic filling-which could improve cardiac performance as well as exercise tolerance at high altitude. While improvements in cardiac function have been reported in patients' taking sildenafil for primary or secondary pulmonary hypertension, the majority of studies using sildenafil in healthy subjects show little, if any, change in cardiac output. ${ }^{9,32,33}$ Moreover, cardiac output/cardiac function is not different during acute hypoxic exercise in healthy subjects treated with a single dose of bosentan (up 
to $250 \mathrm{mg}),{ }^{15}$ thus there seems little chance that reducing RV afterload could improve exercise in normal healthy subjects. ${ }^{19}$ Taken together, these data are consistent with the fact the intrinsic cardiac function is not a limiting factor for cardiac performance during exercise at high altitude and, therefore, improvements in cardiac function contribute very little, if at all, to improved exercise performance in healthy subjects at high altitude.

We were surprised to see that the resting heart rate was significantly elevated in both the sildenafil and bosentan groups (Table 4), especially since most studies at high altitude have not reported the same response..$^{22,23,34}$ But, in fact, ours is not the only study to find an increase in resting heart rate with sildenafil. Ricart et al also report a significant increase in resting heart rate with sildenafil under both normoxia and hypoxia conditions. ${ }^{35}$ But, in contrast to our study, they report no difference in heart rate during hypoxic exercise. ${ }^{35}$ It is interesting to note, however, that Hsu et al have reported healthy individuals as sildenafil "responders" and "nonresponders." 24 In their study, "sildenafil-responders" showed improved cardiac output and exercise performance in hypoxia, whereas "nonresponders" did not. ${ }^{24}$ These data suggest that treating healthy individuals with sildenafil (or similar drugs, such as bosentan) is not likely to be beneficial to everyone, but an explanation for this differential response remains to be determined.

Although we did not measure cardiac output and/or pulmonary artery pressure (PAP) in this study, the effects of sildenafil and bosentan on cardiac output, PVR and PAP have already been well-studied and documented. For example, Ghofrani et al report a $19 \%$ decrease in $\mathrm{PAP}$ at rest and an $18 \%$ decrease in PAP during exercise in healthy mountaineers in a field laboratory located at Mount Everest base camp (5245 m) who were taking the same oral dose of sildenafil $(50 \mathrm{mg})$ we used in this study. ${ }^{22}$ Richalet et al also show sildenafil-induced decreases in PAP (approximately $21-35 \%$ between 2 and 5 days), and report similar improvements in $\mathrm{PaO}_{2}$ with sildenafil after 2 and 5 days at high altitude $(4350 \mathrm{~m}){ }^{23}$ Likewise, Modesti et al report a $32 \%$ decrease in PAP one day after a single dose of bosentan $(62.5 \mathrm{mg})$ at $4559 \mathrm{~m}^{21}$ Thus, there is ample evidence establishing the pulmonary vasodilating effect of these drugs in healthy subjects, and little reason to expect that PAP would not have been similarly reduced in response to sildenafil or bosentan treatment in our subjects.

\section{GENDER DIFFERENCE IN GAS EXCHANGE}

Gender differences in pulmonary structure and function have been well-described. ${ }^{25-28,36-39}$ As expected, women had smaller lung volumes (Table 2) and lower minute ventilation, $\dot{\mathrm{VO}}_{2}$ and $\dot{\mathrm{V}} \mathrm{CO}_{2}$ during exercise compared to men (Table 3). Despite this, we found that women consistently had greater $\mathrm{PaO}_{2}$ and $\mathrm{SaO}_{2}$, at rest and during exercise - both before and after drug treatment-compared to men (Table 3). It is worth noting, however, that $\mathrm{A}-\mathrm{aDO}_{2}$ was not significantly different between men and women, indicating that overall gas exchange was equally impacted by hypoxia in both men and women (Table 3 ). Contrary to recent studies that suggest that women (by virtue of smaller airways, reduced lung size, and/or lower ventilatory response to exercise ${ }^{26,27,40-42}$ ) may be more prone to gas exchange impairment during exercise than men; these data show that young athletic women have at least the same, if not better gas exchange (eg, higher $\mathrm{PaO}_{2}$ and $\mathrm{SaO}_{2}$ ) during hypoxic exercise compared to men. While present findings are consistent with another observation that pulmonary gas exchange during exercise is not worse in women when matched to men according to age-, height- and aerobic capacity, ${ }^{25}$ we would caution against widespread generalization of our results due to the limited number of subjects in our study. Indeed, the principal purpose of this study was to examine gas exchange during hypoxic exercise before and after treatment with sildenafil or bosentan. Using the repeated measures study design, with each subject serving as their own control, provided much greater statistical power (in determining drug effect) than that which can be obtained from a cross-sectional study design (ie, as with ours gender comparison). Further studies with a much greater number of subjects will be needed to make a more compelling cross-sectional comparison in gas exchange between men and women. Nevertheless, it is evident from our data that there was no significant difference in the gas exchange response (between men and women) to the respective drug treatments.

\section{PERSPECTIVE}

There is great concern over ergogenic drugs in many professional and competitive sports arenas. On the other hand, to many amateur athletes and/or active individuals (such as recreational skiers, mountain climbers, outdoor enthusiasts, etc.) these drugs are seen as beneficial because they can minimize and/or alleviate altitude-related problems and improve tolerance of physical activity at high altitude. While the present data suggest that pulmonary vasodilators can improve $\mathrm{PaO}_{2}$, the question relating to whether sildenafil or bosentan may be used (or abused) by healthy individuals in order to achieve better high altitude performance remains uncertain. This is evidenced by the conflicting reports with respect to the benefit of sildenafil on exercise performance at high 
altitude (Table 1). One explanation for variability between these studies may be related to the degree of HPV and/or the level of hypoxemia occurring among individuals. Thus the benefit from any pulmonary vasodilating drug may depend on the degree of pulmonary hypertension induced by hypoxia (or exercise), so that subjects with a greater degree of hypoxia-induced hypertension are likely to achieve the greatest benefit compared to those with a lesser or minimal increase in PAP. This would imply that any benefit from pulmonary vasodilators on pulmonary gas exchange may only be realized in the presence of pulmonary arterial hypertension, which could-at least in part-explain the varied responses to exercise performance currently reported in the literature (Table 1) and why some individuals respond to treatment and others do not. While there is evidence that sildenafil treatment can improve physical performance in some healthy individuals under hypoxic conditions, the notion that similar benefits might occur in healthy subjects taking sildenafil under normoxic (ie, sea level) conditions is not supported. ${ }^{24}$

\section{Conclusion}

These data show that sildenafil and bosentan, both of which are clinically used to reduce PAP in patient populations with pulmonary hypertension, are capable of improving pulmonary gas exchange in young healthy (male and female) subjects exercising in hypoxia. It is be tempting to speculate that the improvements in oxygenation we observed in our healthy subjects might translate to improved physical performance at high altitude. However, it must be emphasized that our study was not designed to address this question, and evidence of improved exercise capacity in healthy individuals taking sildenafil or bosentan is limited and remains equivocal at best. Nonetheless, these data suggest that sildenafil and bosentan can improve $\mathrm{PaO}_{2}$ during hypoxic exercise. Even though the improvement was small $(2-3 \mathrm{mmHg})$, it is likely to be physiologically important given the degree of hypoxemia and the energy demands encountered during hypoxic exercise.

\section{References}

1. Gale G, Torre-Bueno J, Moon R, Saltzman H, Wagner P. Ventilation-perfusion inequality in normal humans during exercise at sea-level and simulated altitude. J Appl Physiol. 1985;58:978-988.

2. Torre-Bueno J, Wagner P, Saltzman H, Gale G, Moon R. Diffusion limitation in normal humans during exercise at sea level and simulated altitude. J Appl Physiol. 1985;58: 989-995.
3. Hopkins SR, Gavin TP, Siafakas NM, et al. Effect of prolonged, heavy exercise on pulmonary gas exchange in athletes. J Appl Physiol. 1998;85:1523-1532.

4. Podolsky A, Eldridge MW, Richardson RS, et al. Exerciseinduced VA/Q inequality in subjects with prior highaltitude pulmonary edema. J Appl Physiol. 1996;81: 922-932.

5. Hackett P, Rennie D. High-altitude pulmonary edema. JAMA. 2002;287:2275-2278.

6. Swenson ER, Maggiorini M, Mongovin S, et al. Pathogenesis of high-altitude pulmonary edema: inflammation is not an etiologic factor. JAMA. 2002;287:2228-2235.

7. Voelkel NF. High-altitude pulmonary edema. $N$ Engl J Med. 2002;346:1606-1607.

8. Galiè N, Ghofrani HA, Torbicki A, et al. Sildenafil citrate therapy for pulmonary arterial hypertension. $N$ Engl J Med. 2005;353:2148-2157.

9. Sastry BK, Narasimhan C, Reddy NK, Raju BS. Clinical efficacy of sildenafil in primary pulmonary hypertension: a randomized, placebo-controlled, double-blind, crossover study. J Am Coll Cardiol. 2004;43:1149-1153.

10. Suntharalingam J, Treacy CM, Doughty NJ, et al. Longterm use of sildenafil in inoperable chronic thromboembolic pulmonary hypertension. Chest. 2008;134:229-236.

11. Zhao L, Mason NA, Morrell NW, et al. Sildenafil inhibits hypoxia-induced pulmonary hypertension. Circulation. 2001;104:424-428.

12. Channick R, Badesch DB, Tapson VF, et al. Effects of the dual endothelin receptor antagonist bosentan in patients with pulmonary hypertension: a placebo-controlled study. J Heart Lung Transplant. 2001;20:262-263.

13. Galiè N, Hinderliter AL, Torbicki A, et al. Effects of the oral endothelin-receptor antagonist bosentan on echocardiographic and doppler measures in patients with pulmonary arterial hypertension. J Am Coll Cardiol. 2003;41: 1380-1386.

14. Liu C, Chen J. Endothelin receptor antagonists for pulmonary arterial hypertension. Cochrane Database Syst Rev. 2006;3:CD004434.

15. Pham I, Wuerzner G, Richalet JP, Peyrard S, Azizi M. Endothelin receptors blockade blunts hypoxia-induced increase in PAP in humans. Eur J Clin Invest. 2010;40: 195-202.

16. Ghofrani HA, Pepke-Zaba J, Barbera JA, et al. Nitric oxide pathway and phosphodiesterase inhibitors in pulmonary arterial hypertension. J Am Coll Cardiol. 2004 Jun 16; 43(Supp112):68-72.

17. Rich S. The current treatment of pulmonary arterial hypertension: time to redefine success. Chest. 2006;130: 1198-1202.

18. Faoro V, Boldingh S, Moreels M, et al. Bosentan Decreases Pulmonary Vascular Resistance and Improves Exercise Capacity in Acute Hypoxia. Chest. 2009[Epub ahead of print].

19. Faoro V, Lamotte M, Deboeck G, et al. Effects of sildenafil on exercise capacity in hypoxic normal subjects. High Alt Med Biol. 2007;8:155-163. 
20. Kleinsasser A, Loeckinger A. Are sildenafil and theophylline effective in the prevention of high-altitude pulmonary edema? Med Hypotheses. 2002;59:223-225.

21. Modesti PA, Vanni S, Morabito M, et al. Role of endothelin-1 in exposure to high altitude: Acute Mountain Sickness and Endothelin-1 (ACME-1) study. Circulation. 2006;114:1410-1416.

22. Ghofrani HA, Reichenberger F, Kohstall MG, et al. Sildenafil increased exercise capacity during hypoxia at low altitudes and at Mount Everest base camp: a randomized, double-blind, placebo-controlled crossover trial. Ann Intern Med. 2004;141:169-177.

23. Richalet JP, Gratadour P, Robach P, et al. Sildenafil inhibits altitude-induced hypoxemia and pulmonary hypertension. Am J Respir Crit Care Med. 2005;171:275-281.

24. Hsu AR, Barnholt KE, Grundmann NK, Lin JH, McCallum SW, Friedlander AL. Sildenafil improves cardiac output and exercise performance during acute hypoxia, but not normoxia. J Appl Physiol. 2006;100:2031-2040.

25. Olfert IM, Balouch J, Kleinsasser A, et al. Does gender affect human pulmonary gas exchange during exercise? J Physiol. 2004;557:529-541.

26. Harms C, McClaran S, Nickele G, Pegelow D, Nelson W, Dempsey J. Exercise-induced arterial hypoxemia in healthy young women. J Physiol. 1998;507:619-628.

27. Hopkins SR, Harms CA. Gender and pulmonary gas exchange during exercise. Exerc Sport Sci Rev. 2004;32: 50-56.

28. Ricart A, Pages T, Viscor G, Leal C, Ventura JL. Sexlinked differences in pulse oxymetry. Br J Sports Med. 2008;42:620-621.

29. Snyder EM, Beck KC, Hulsebus ML, Breen JF, Hoffman EA, Johnson BD. Short Term Hypoxic Exposure at Rest and During Exercise Reduces Lung Water in Healthy $\mathrm{Hu}-$ mans. J Appl Physiol. 2006;101:1623-1632.

30. Kleinsasser A, Loeckinger A, Hoermann C, et al. Sildenafil modulates hemodynamics and pulmonary gas exchange. Am J Respir Crit Care Med. 2001;163:339-343.
31. Blanco I, Gimeno E, Munoz PA, et al. Hemodynamic and gas exchange effects of sildenafil in patients with chronic obstructive pulmonary disease and pulmonary hypertension. Am J Respir Crit Care Med. 2010;181:270-278.

32. Hirata K, Adji A, Vlachopoulos C, O'Rourke MF. Effect of sildenafil on cardiac performance in patients with heart failure. Am J Cardiol. 2005;96:1436-1440.

33. Noori S, Friedlich P, Wong P, Garingo A, Seri I. Cardiovascular effects of sildenafil in neonates and infants with congenital diaphragmatic hernia and pulmonary hypertension. Neonatology. 2007;91:92-100.

34. Cornolo J, Mollard P, Brugniaux JV, Robach P, Richalet JP. Autonomic control of the cardiovascular system during acclimatization to high altitude: effects of sildenafil. J Appl Physiol. 2004;97:935-940.

35. Ricart A, Maristany J, Fort N, Leal C, Pagés T, Viscor G. Effects of sildenafil on the human response to acute hypoxia and exercise. High Alt Med Biol. 2005;6:43-49.

36. Thurlbeck WM. Postnatal human lung growth. Thorax. 1982;37:564-571.

37. Guenette JA, Witt JD, McKenzie DC, Road JD, Sheel AW. Respiratory mechanics during exercise in endurancetrained men and women. J Physiol. 2007;581:1309-1322.

38. Guenette JA, Diep TT, Koehle MS, Foster GE, Richards JC, Sheel AW. Acute hypoxic ventilatory response and exercise-induced arterial hypoxemia in men and women. Respir Physiol Neurobiol. 2004;143:37-48.

39. Mead J. Dysanapsis in normal lungs assessed by the relationship between maximal flow, static recoil, and vital capacity. Am Rev Respir Dis. 1980;121:339-342.

40. Sheel AW, Richards JC, Foster GE, Guenette JA. Sex differences in respiratory exercise physiology. Sports Med. 2004;34:567-579.

41. Sheel AW, Guenette JA. Mechanics of breathing during exercise in men and women: sex versus body size differences? Exerc Sport Sci Rev. 2008;36:128-134.

42. Guenette JA, Sheel AW. Exercise-induced arterial hypoxaemia in active young women. Appl Physiol Nutr Metab. 2007;32:1263-1273. 